\title{
P308
}

\section{Repeatability of Land Time-lapse Seismic Surveys - Otway Project 2D Test Line Case Study}

\author{
R. Pevzner* (Curtin University of Technology), V. Shulakova (CSIRO \\ Petroleum), A. Kepic (Curtin University of Technology), Y.Y.M. Al-Jabri \\ (Curtin University of Technology) \& M. Urosevic (Curtin University of \\ Technology)
}

\section{SUMMARY}

Full range of 2D and 3D borehole and surface seismic methods are utilised for time-lapse surveys for monitoring of $\mathrm{CO} 2$ sequestration at Otway Basin pilot project site. Conducting land time-lapse surveys is challenging task because of typical variability of ground conditions, source-receiver coupling, ambient noise which results in poor repeatability of land seismic data. We analysed the major factors influencing repeatability of land seismic data. We also analysed both synthetic and field data for possible limitations of the surface reflection seismic method when it comes to repeatability.

We show that changes in near surface conditions will produce kinematic differences but also different ground roll patterns. This will in general require slightly different parameters for processing of two successive surveys if they are acquired for vastly different soil conditions. We then conduct extensive numerical and field tests to show that the $\mathrm{S} / \mathrm{N}$ variability as function of the source strength relative to the background noise level is crucial. Source type is less important for time lapse surveys as long as $\mathrm{S} / \mathrm{N}$ ratio is high. Our tests included impact (weight drop) and vibrating (IVI Mini-Buggy) sources. 


\section{Introduction}

A time-lapse seismic is a method of choice for monitoring changes in a reservoir related to oil/gas reservoir depletion or $\mathrm{CO}_{2}$ sequestration. However conducting land time-lapse surveys is a challenging task because of typically poor repeatability of land seismic data. Understanding factors influencing repeatability and possible limitations of the surface reflection seismic method is crucially important for success of this kind of surveys.

A unique set of repeated 2D test surveys was collected over last three years within scientific program of $\mathrm{CO} 2 \mathrm{CRC}$ Otway pilot project, Australia. This includes 7 repeated surveys obtained with same geometry but different sources (several different weight drops and vibrators) and near-surface conditions varying from survey to survey due to changes in soil saturation.

In this paper we selected 3 repeated surveys to determine key limiting factors affecting repeatability of land seismic surveys:

1. 2008, dry season, weight drop source data

2. 2008, dry season, IVI Mini-Buggy source data

3. 2007, wet season, weight drop data

This set of surveys would allow us to compare pairs of 2D seismic lines acquired with: 1) Same nearsurface conditions, but with different sources (2008 data); 2) Different near-surface conditions, but with similar sources (two weight drop surveys); 3) Acquired with different near-surface conditions, and with different sources.

\section{Acquisition and processing parameters}

Acquisition parameters are presented in table 1.

Table 1: Acquisition parameters

\begin{tabular}{|c|c|c|c|}
\hline & Test1 & Test2 & Test3 \\
\hline Source Type & Weight Drop & Weight Drop & Mini Buggy \\
\hline Date & June, 2007 & $\begin{array}{l}\text { November, } \\
2008\end{array}$ & $\begin{array}{l}\text { November, } \\
2008\end{array}$ \\
\hline Weather condition & Wet & Dry & Dry \\
\hline Total Number of Sources & 158 & 155 & 155 \\
\hline $\begin{array}{l}\text { Total Number of } \\
\text { Receivers }\end{array}$ & 162 & 156 & 159 \\
\hline $\begin{array}{l}\text { Source/Receiver Point } \\
\text { Spacing, m }\end{array}$ & $10 / 10$ & $10 / 10$ & $10 / 10$ \\
\hline Number of Channels & 162 & 156 & 159 \\
\hline Offset range, $m$ & $5-1605$ & $5-1545$ & $5-1545$ \\
\hline Reference Character & WD 2007 & WD 2008 & MB 2008 \\
\hline
\end{tabular}

Receiver and source points were located at the same position (source points between receiver points) along same line for all surveys. Once receivers were fixed they were not moved during a survey. Length of the record for weight drops survey was $3 \mathrm{~s}, 1 \mathrm{~ms}$ sampling interval, Mini-Buggy survey recorded with $10-120 \mathrm{~Hz}$ liner $12 \mathrm{~s}$ sweep, $15 \mathrm{~s}$ recording time.

Processing of the data was performed with ProMax (Landmark, Halliburton) and RadExPro (DECO Geophysical) processing software systems. We tried to use similar amplitude-preserving processing flows for all three datasets, however it was found to be impossible to use exactly the same flows. There are two main reasons for that. First, variation in near surface conditions resulted in static corrections being of different magnitude and consequently stacking velocities were different for the two surveys. Next is that changes in near surface conditions and/or properties of the source affects properties of coherent noises, such as ground roll.

Several routines were applied to cross-equalize the data. At first stage post-stack static shifts were applied to match reflectors geometry on different surveys. Data, obtained in 2008 with IVI MiniBuggy source is selected as a reference dataset due to relatively high quality. Two other surveys were processed to match this dataset.

To arrive to a common wavelet (i.e. amplitude and phase spectra) for repeated surveys a single shaping filter (Wiener) was designed for each pair of surveys by averaging of all shaping filters (100 samples, $0.1 \%$ of random noise) computed for each pair of corresponding traces. 


\section{0 \\ Amsterdam 'o9}

\section{Repeatability analysis of stacked sections}

Cross equalized sections and difference between them for all three pairs of repeated surveys were computed. An example of such comparison for surveys acquired in 2007 and 2008 with weight drop source is presented in Figure 1. Normalized RMS values and $\mathrm{S} / \mathrm{N}$ estimations were computed along 5 horizons, as shown in Figure1, using time window $50 \mathrm{~ms}$ around each horizon). Only full fold section (CDP range 40-260) was included into analysis.



Figure 1. WD 2007 wet and WD 2008 dry stacked section comparison

To compute NRMS we used following equation (Kragh. E. and Christie, P. A. F., 2002):

$$
N R M S=200 \% \frac{R M S(a-b)}{R M S(a)+R M S(b)},
$$

where $a$ and $b$ are two surveys being compared. Signal to noise ratio was estimated between pairs of consecutive traces on each survey in same time windows as NRMS value using equation (Hatton et al. 1986) (2).

$$
S N_{i}=\sqrt{\frac{\left[g_{i, i+1}\right]_{M A X}}{1-\left[g_{i, i+1}\right]_{M A X}}}
$$

where $i$ is trace number, $g_{i, i+1}$ is normalized cross-correlation function between $i$ and $i+1$ traces and $\left[g_{i, i+1}\right]_{\text {MAX }}$ is its maximum value. This equation allows us to obtain signal/noise ratio in assumption what noise is additive, uncorrelated and have zero mean value.

Since Waarre $\mathrm{C}$ horizon is almost not visible on sections other than one obtained with IVI MiniBuggy due to insufficient power of the source it is included into this survey only to demonstrate how repeatability can be improved by increasing of $\mathrm{S} / \mathrm{N}$ ratio.

In general it is possible to conclude (Figure 2) that non-repeatability between data acquired with same surface conditions (WD 2008 vs MB 2008, Figure 2B, blue bars) is less than between WD surveys themselves acquired over two different seasons. So near-surface condition is most likely one of the factors of the primary importance. Also one can observe that the highest non-repeatability is observed between the pair of surveys (WD 2008 vs WD 2007, green bars), obtained with same source but in different years, under dry and wet near surface conditions, respectively. Thus the source change accounts for secondary effect only when it comes to repeatability. 


\section{Amsterdam 'o9}
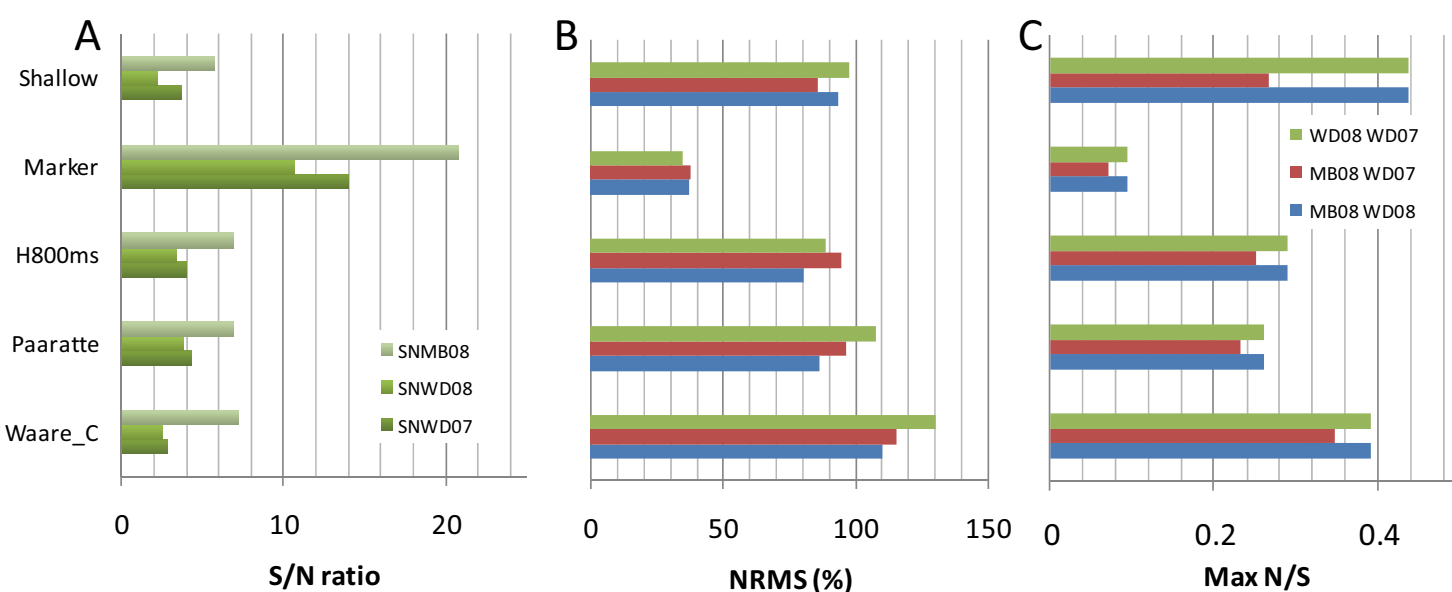

Figure 2: Signal to noises $/ N$ ) ratio for data acquired with different sources computed over several horizons: (A) $S / N$ ratio, (B) NRMS and (C) maximum noise-to-signal N/S. All values computed for different pairs of surveys.

Interestingly there is no considerable difference between non-repeatability computed for different combinations of surveys. To determine key issue of differences between datasets we need to consider signal/noise ratio plots. Comparing the two figures (Figure 2A and B) it is obvious that horizons with greater $\mathrm{S} / \mathrm{N}$ ratio correspond to those with higher repeatability. To prove this statement we selected $\mathrm{S} / \mathrm{N}$ value from each pair of surveys being analysed for each horizon and compared inverse N/S (noise/signal) to NRMS corresponded values (Figure 2C). One can observe practically linear relationship between N/S and NRMS computed values! Subsequently we used a simple mathematical modelling to determine principal limitation of NRMS measure due to presence of non-repeatable random noise.

\section{Contribution of ambient noise to limitation of repeatability}

We use the following workflow to investigate relationship between S/N and NRMS:

1. Synthetic seismic section consisted of 120 traces with only one horizontal event computed (we use Ormsby wavelet, 10-20-60-80 Hz).

2. To produce a pair of 'baseline' and 'repeated' survey with needed S/N ratio, variable amount of random noise with the same bandwidth as signal $(10-20-60-80 \mathrm{~Hz})$ was introduced into synthetic sections. An example of 'noisy' section is presented in Figure 3A.

3. $\mathrm{S} / \mathrm{N}$ ratio and NRMS value computed in $50 \mathrm{~ms}$ time window along the event using same approach as applied to real data

4. Steps 2 and 3 repeated several times with changing amount of random noise introduced into seismic section.

A)

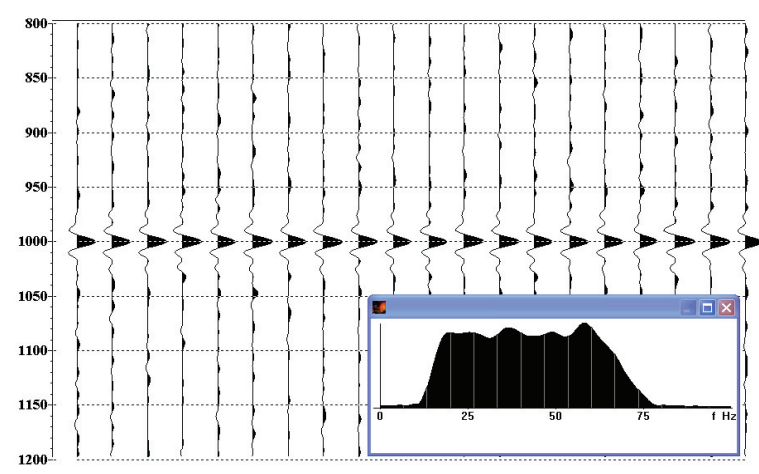



Figure 3: Example of a 'noisy' seismogram (A) and measured versus synthetic S/N - NRMS relationship (B). 




Result of this modelling is presented in Figure 3B. Red points represents pairs of NRMS and minimum $\mathrm{S} / \mathrm{N}$ (i.e. noisiest survey in the each pair is controlling repeatability). This plot demonstrates that is possible to achieve NRMS value of less than $40 \%$ if $\mathrm{S} / \mathrm{N}$ ratio at the target horizons is higher than 10. In fact if successive surveys were measured with the same soil conditions and using the same source it is possible to keep non-repeatability close to $20 \%$.

\section{Influence of near-surface conditions on repeatability}

Near surface agricultural layer combined with the rugged limestone surface (which constitutes a nearsurface part of the section in the Otway basin) is likely to create scattering which will change with soil

saturation that is underground water level. Top soil hardness will be also more important for impact sources such as weight drop. To get the idea of the magnitude of these effects onto non-repeatability we conducted a series of numerical tests (Al-Jabri et al., 2008) there we compared stacked sections with different models of near-surface properties obtained by finite-difference modelling.

\section{Conclusions}

Processing experience and repeatability analysis allows us to make following conclusions:

1. Changes in near surface conditions due to variable soil saturation will produce kinematic effects (changes in statics, stacking velocities) and produce different ground roll patterns. In general it seems to be impossible to apply same processing sequence (with same parameters of each routine) to repeated land surveys which were acquired in different time of the year.

2. Repeatability analysis was carried out across different reflectivity patterns, time and depth intervals. It is clear that the main factor affecting repeatability is low signal-to-noise ratio (i.e. the power of seismic source used during acquisition, relative to the background noise level). In case of comparison of two different surveys with different $\mathrm{S} / \mathrm{N}$ ratios NRMS will be controlled by the difference in source strength, signal to coherent and background noise levels and the similarity in frequency content of the sources. Overall the $\mathrm{S} / \mathrm{N}$ variability as function of the source strength relative to the background noise level is crucial. Source type is less important.

3. IVI Mini-Buggy provides data with $\mathrm{S} / \mathrm{N}$ ratio twice bigger than the one produced with weight drops. Hence it is preferred source for time-lapse 3D surveys in Otway basin. To achieve NRMS $40-50 \% \mathrm{~S} / \mathrm{N}$ must be approximately equals to 10 . Taking into account greater fold of 3D survey and better illumination of the target than that achieved with these $2 \mathrm{D}$ tests, repeatability goal is achievable with IVI Mini-Buggy source.

\section{Acknowledgement}

This work is sponsored by CO2CRC and we thank S. Sharma for making these experiments possible. We are grateful to our colleagues from Curtin University C. Dupuis and A. Dzunic for motivating discussions and help with data acquisition.

\section{References}

Al-Jabri, Y.Y.M., Urosevic, M and Sherlock, D. [2008] The Effect of Corrugated Limestone and the Changing of the Near Surface Conditions on CO2 Monitoring Program at Naylor-1, CO2CRC Otway Project, Victoria, Australia. First EAGE CO2 Geological Storage Workshop, Extended Abstracts, A12

Hatton, L., Worthington, M. H. and Makin, J. [1986] Seismic Data Processing: Theory and Practice. Oxford, UK: Blackwell Scientific Publications, p. 177.

Kragh. E. and Christie, P. A. F. [2002] Seismic repeatability, normalized RMS, and predictability, The Leading Edge, 21(7), 640-647. 